\title{
ON HOMOLOGICAL FROBENIUS COMPLEXES AND BIMODULES
}

\author{
J. R. GARCÍA ROZAS, LUIS OYONARTE AND BLAS TORRECILLAS \\ Departamento de Matemáticas, Universidad de Almería, 04071 Almería, Spain \\ e-mails: \{jrgrozas, oyonarte, btorreci\}@ual.es
}

(Received 25 April 2013; revised 22 July 2013; accepted 31 July 2013)

\begin{abstract}
We introduce the concept of homological Frobenius functors as the natural generalization of Frobenius functors in the setting of triangulated categories, and study their structure in the particular case of the derived categories of those of complexes and modules over a unital associative ring. Tilting complexes (modules) are examples of homological Frobenius complexes (modules). Homological Frobenius functors retain some of the nice properties of Frobenius ones as the ascent theorem for Gorenstein categories. It is shown that homological Frobenius ring homomorphisms are always Frobenius.
\end{abstract}

2010 Mathematics Subject Classification. Primary 16E65. Secondary 18E30.

1. Introduction. The notion of Frobenius adjoint pair between categories of modules was introduced by Morita in [15] as the most natural setting for the study of Frobenius extensions. A pair of adjoint functors $(L, R)$ between two module categories is said to be Frobenius if $R$ is also a left adjoint of $L$. This theory was later extended to the arbitrary Grothendieck categories (see [3]), so the study of the Frobenius property for categories of graded rings, comodules over a coring and other important Grothendieck categories (see [2]) became a natural question. Due to its applications to the theory of Hopf algebras, monoidal categories and two-dimensional topological quantum field theory, the theory of Frobenius extensions has recently been a subject of increasing interest (see for example [10]). In this paper, we introduce the notion of Frobenius adjoint pair between two triangulated categories, so in particular derived categories of modules categories.

Frobenius pair of functors can be considered as an extension of Morita equivalences, so our theory generalizes, in the same direction, the theory of equivalences between derived categories of modules, i.e. tilting theory. Then we introduce the notion of homological Frobenius complexes and homological Frobenius bimodules.

The paper is organized as follows. In Section 3 we give the definition of a Frobenius adjoint pair of functors between triangulated categories, in particular, between derived categories. We prove several interesting properties verified by this functors, for instance, under some conditions if we have such a pair $(F, G), F: \mathcal{C} \rightarrow \mathcal{D}$, and $\mathcal{C}$ is Gorenstein, then $\mathcal{D}$ is Gorenstein.

Section 4 is devoted to the study of homological Frobenius complexes of bimodules. These kind of complexes induce Frobenius adjoint pairs in the derived categories of the respective module categories. The examples show that these complexes are close to be tilting complexes of bimodules, but, in general, they are not. We also 
show that the endomorphism Differential Graded (DG)-algebra of a homological Frobenius complex is a Frobenius extension with respect to the base ring.

In Section 5 we study homological Frobenius bimodules and extensions. We prove that when the homological Frobenius bimodule ${ }_{S} M_{R}$ is projective on one side then it is a Frobenius bimodule. This implies that homological Frobenius ring homomorphisms are always classical Frobenius extensions. We also prove that any homological Frobenius adjoint pair between module categories is given by a homological Frobenius bimodule. We relate totally reflexive extensions with the Frobenius ones. Finally, we generalize Enochs' result about Gorenstein ascent in Frobenius ring extensions.

2. Preliminaries. Triangulated categories will be used occasionally along the paper, as well as triangulated functors and related concepts. We refer to Neeman's book [17] for definitions and terminology about these topics. However, we recall that an object $C$ in a triangulated category $\mathcal{T}$ is said to be compact if $\operatorname{Hom}_{\mathcal{T}}(C,-)$ commutes with arbitrary coproducts. Also, a triangulated category with arbitrary coproduct $\mathcal{T}$ is compactly generated if there exists a set of compact objects $\mathcal{A}$ with the following property: If $\operatorname{Hom}_{\mathcal{T}}(A, X)=0$ for each $A \in \mathcal{A}$, then $X=0$.

Let $\mathcal{C}$ be an abelian category and $\mathbf{C}(\mathcal{C})$ be the category of complexes in $\mathcal{C}$. By $\mathbf{K}(\mathcal{C})$ we denote the homotopy category associated with $\mathcal{C}$. We follow the standard notation $\mathbf{K}^{b}(\mathcal{C}), \mathbf{K}^{+}(\mathcal{C}), \mathbf{K}^{-}(\mathcal{C})$ for bounded, bounded below and bounded above complexes respectively. If $\mathcal{T} \subset \mathcal{C}$ is a full subcategory, then we denote by $\mathbf{K}^{*}(\mathcal{T})$ the full triangulated subcategory of $\mathbf{K}^{*}(\mathcal{C})(*=b,+,-)$ of complexes with components in $\mathcal{T}$. We also denote by $\mathbf{C}^{+, b}(\mathcal{T})$ the full subcategory of $\mathbf{C}^{+}(\mathcal{T})$ of complexes with a finite number of non-zero group cohomologies.

If $X$ is an object of $\mathcal{C}$, we write $X[i]$ to denote the complex with $X$ in the $i$ th component and zero in the rest of components. Let $\operatorname{add}(X)$ be the full subcategory of $\mathcal{C}$ of direct summand of finite direct sums of copies of $X$.

The derived category of $\mathcal{C}$, denoted as $\mathbf{D}(\mathcal{C})$, is the localization of $\mathbf{K}(\mathcal{C})$ by a set of representatives of all quasi-isomorphisms. Similarly, we define $\mathbf{D}^{*}(\mathcal{C})(*=b,+,-)$ as the localization of $\mathbf{K}^{*}(\mathcal{C})(*=b,+,-)$. We let $Q^{*}: \mathbf{K}^{*}(\mathcal{C}) \rightarrow \mathbf{D}^{*}(\mathcal{C})$ be the respective localization maps.

Suppose $\mathcal{C}$ is a Grothendieck category, so it has enough injectives. It is well know that given a complex $X \in \mathbf{C}(\mathcal{C})$ there exists an exact sequence of complexes:

$$
0 \rightarrow X \rightarrow I \rightarrow E \rightarrow 0
$$

where $E$ is acyclic and $I$ is DG-injective (see $[\mathbf{8}, \mathbf{1 8}, \mathbf{1 9}]$ ). This implies that $X=I$ in $\mathbf{D}(\mathcal{C})$. We call $\mathbf{i} X=I$, an injective resolution of $X$. Note that if $X \in \mathbf{C}^{+}(\mathcal{C})$, then there is an injective resolution $I \in \mathbf{C}^{+}(\mathcal{C})$ with $X=I$ in $\mathbf{D}^{+}(\mathcal{C})$. Then $\mathbf{D}(\mathcal{C}) \cong \mathbf{K}(\mathcal{D} G-I)$ as triangulated categories where $\mathcal{D} G-I$ is a full subcategory of $\mathrm{DG}$-injective complexes. In particular, since any bounded below complex of injectives is DG-injective, it follows that $\mathbf{D}^{+}(\mathcal{C}) \cong \mathbf{K}^{+}(\mathcal{I})$, where $\mathcal{I}$ is a full subcategory of complexes with injective components. As a consequence, we also get the triangulate equivalence $\mathbf{D}^{b}(\mathcal{C}) \cong \mathbf{K}^{b}(\mathcal{I})$.

Dually, if $\mathcal{C}$ is a Grothendieck category with enough projectives, there exists an exact sequence of complexes:

$$
0 \rightarrow E^{\prime} \rightarrow P \rightarrow X \rightarrow 0
$$

where $E^{\prime}$ is acyclic and $P$ is DG-projective $([\mathbf{8}, \mathbf{1 8}, \mathbf{1 9}])$. Thus, $X=P$ in $\mathbf{D}(\mathcal{C})$. We call $\mathbf{p} X=P$ a projective resolution of $X$. If $X \in \mathbf{C}^{-}(\mathcal{C})$, then there is a projective 
resolution $P \in \mathbf{C}^{-}(\mathcal{C})$ with $X=P$ in $\mathbf{D}^{-}(\mathcal{C})$. Dually to the DG-injective case, $\mathbf{D}(\mathcal{C}) \cong$ $\mathbf{K}(\mathcal{D} G-P)$ as triangulated categories, where $\mathcal{D} G-P$ is a full subcategory of DGprojective complexes. In particular, since any bounded above complex of projectives is DG-projective, it follows that $\mathbf{D}^{-}(\mathcal{C}) \cong \mathbf{K}^{-}(\mathcal{P})$, where $\mathcal{P}$ is a full subcategory of complexes with projective components. Also, $\mathbf{D}^{b}(\mathcal{C}) \cong \mathbf{K}^{b}(\mathcal{P})$.

Let $\mathcal{C}$ and $\mathcal{D}$ be two Grothendieck categories and suppose $\mathcal{C}$ has enough projectives. Let $F: \mathbf{C}(\mathcal{C}) \rightarrow \mathbf{C}(\mathcal{D})$ be a right exact functor and $G: \mathbf{C}(\mathcal{D}) \rightarrow \mathbf{C}(\mathcal{C})$ be a left exact functor. Their left and right derived functors will be denoted by $\mathbf{L} F$ and $\mathbf{R} G$ respectively, so $\mathbf{L} F: \mathbf{D}(\mathcal{C}) \rightarrow \mathbf{D}(\mathcal{D})$ and $\mathbf{R} G: \mathbf{D}(\mathcal{D}) \rightarrow \mathbf{D}(\mathcal{C})$ are defined as $\mathbf{L} F(X)=F(\mathbf{p} X)(\mathbf{p} X$ is a projective resolution of $X), \mathbf{R} G(Y)=G(\mathbf{i} Y)(\mathbf{i} Y$ is an injective resolution of $Y)$. If $(F, G)$ is an adjoint situation then $F$ preserves arbitrary colimits and $G$ preserves arbitrary limits and the pair $(\mathbf{L} F, \mathbf{R} G)$ is an adjoint pair of functors between triangulated categories.

Recall that a Grothendieck category is said to be Gorenstein [6], provided that the following conditions hold:

(1) An object is of finite projective dimension if and only if it is of finite injective dimension.

(2) Both finitistic projective and injective dimensions (FPD and FID respectively) of the category are finite.

(3) The category has a generator of finite projective dimension.

We note that any Grothendieck category has enough injectives. Therefore, we can compute $\operatorname{Ext}^{i}(X, Y)$ using an injective resolution of $Y$. Thus, the projective dimension of an object $X$ is $\leq n$ if and only if $\operatorname{Ext}^{i}(X,-)=0 \forall i>n$, so we do not need projectives to define the projective dimension.

A Gorenstein category is said to be $n$-Gorenstein provided that both $F I D$ and $F P D$ are $\leq n$.

Let $R$ be a ring. The category of all unitary left $R$-modules is denoted by $R$-Mod. Let $M^{\bullet}$ and $N^{\bullet}$ be two complexes of left $R$-modules. By $\operatorname{Hom}^{\bullet}\left(M^{\bullet}, N^{\bullet}\right)$ we denote the Hom-complex. In case $N$ is concentrated in one degree we just write $\operatorname{Hom}_{R}\left(M^{\bullet}, N\right)$.

For a complex of left $R$-modules $M^{\bullet}$ and a complex of right $R$-modules $L^{\bullet}$, we denote by $M^{\bullet} \otimes_{R}^{\bullet} N^{\bullet}$ the usual tensor complex. The $i$ th shift of a complex $M^{\bullet}$ will be denoted by $M^{\bullet}[i]$.

When there is no confusion about the side of $R$-modules, we simply write $\mathbf{D}(R)$ instead of $\mathbf{D}(R-M o d)$. In the triangulated category $\mathbf{D}(R)$ the compact objects are well known: they are the so-called perfect complexes (bounded complexes of finitely generated projective components).

A complex of right $R$-modules $T_{R}^{\bullet}$ is a tilting complex if it is a perfect complex, it generates the full subcategory formed by compact objects and $\operatorname{Hom}_{\mathbf{D}(R)}\left(T^{\bullet}, T^{\bullet}[i]\right)=0$ for all $i \in \mathbb{Z}$. If we let $S=\operatorname{End}_{\mathbf{D}(R)}(T)$, then $T^{\bullet}$ gives the existence of an equivalence between the derived categories $\mathbf{D}(R)$ and $\mathbf{D}(S)[\mathbf{1 4}]$.

A DG-algebra over $R$ is a $\mathbb{Z}$-graded $R$-algebra $A=\oplus_{n \in \mathbb{Z}} A^{n}$ that is also a complex over $R$ that satisfies Leibniz's Rule:

$$
\partial(a b)=(\partial a) b+(-1)^{|a|} a(\partial b) \forall a, b \in A .
$$

Equivalently, a DG-algebra over $R$ is a $\mathbb{Z}$-graded $R$-algebra $A$ with a squarezero derivation $\partial: A \rightarrow A$ of degree 1 . If $M^{\bullet}$ is a complex of $R$-modules, then $\operatorname{Hom}^{\bullet}\left(M^{\bullet}, M^{\bullet}\right)$ is a DG-algebra over $R$. 
Given DG-algebras $A$ and $B$ over $R$, a morphism of DG-algebras $f: A \rightarrow B$ is a morphism of complexes for which $f\left(1_{A}\right)=1_{B}$ and $f\left(a_{1} a_{2}\right)=f\left(a_{1}\right) f\left(a_{2}\right)$ $\forall a_{1}, a_{2} \in A$.

A DG- $A$-module is a graded module $M$ over the graded algebra $A$ that is also a complex that satisfies Leibniz's Rule:

$$
\partial(a m)=(\partial a) m+(-1)^{|a|} a(\partial m) \forall a \in A, \forall m \in M .
$$

Let ${ }_{S} M_{R}$ be an $(S, R)$-bimodule. We say that $M$ is a Frobenius bimodule if ${ }_{S} M$ and $M_{R}$ are projective and there is an isomorphism of $(R, S)$-bimodules $\operatorname{Hom}_{R}(M, R) \cong$ $\operatorname{Hom}_{S}(M, S)$ (see [3]). Given a ring homomorphism $\psi: R \rightarrow S$, we say that $\psi$ is a Frobenius homomorphism if ${ }_{S} S_{R}$ is a Frobenius $(S, R)$-bimodule. In particular, we have Frobenius extensions of rings $R \subseteq S$.

\section{Homological Frobenius functors and Gorenstein categories.}

Definition 3.1. Let $\mathcal{T}$ and $\mathcal{S}$ be two triangulated categories and $F: \mathcal{T} \rightarrow \mathcal{S}$ an exact functor. We say that $F$ is a Frobenius functor if there is an exact functor $G: \mathcal{S} \rightarrow \mathcal{T}$ such that $(F, G)$ and $(G, F)$ are adjoint pairs of functors.

In this case we also say that $(F, G)$ is a Frobenius pair.

Definition 3.2. Let $\mathcal{C}$ and $\mathcal{D}$ be two Grothendieck categories. We say that the couple of adjoint (covariant) functors $(F, G), F: \mathcal{C} \rightarrow \mathcal{D}$ is a homological Frobenius pair if the couple $(\mathbf{L} F, \mathbf{R} G), \mathbf{L} F: \mathbf{D}(\mathcal{C}) \rightarrow \mathbf{D}(\mathcal{D})$ is a Frobenius pair. In this case we say that $F$ and $G$ are homological Frobenius functors.

Lemma 3.3. Let $F: \mathcal{C} \rightarrow \mathcal{D}$ be a homological Frobenius functor. The following statements hold:

(i) $\mathbf{L} F$ preserves products and coproducts.

(ii) If $\mathbf{D}(\mathcal{C})$ and $\mathbf{D}(\mathcal{D})$ are compactly generated, then $\mathbf{L} F$ preserves compact objects.

Proof. (i) $\mathbf{L} F$ has left and right adjoints.

(ii) Follows by [16, Theorem 5.1].

REMARK 3.4. Let $\mathcal{C}$ and $\mathcal{D}$ be two Grothendieck categories. Consider the category $h \operatorname{Fr}(\mathcal{C}, \mathcal{D})$ of homological Frobenius functors $F, G: \mathcal{C} \rightarrow \mathcal{D}$ as objects and natural transformations $F \Rightarrow G$ as morphisms. By [3, Lemma 5.1], $h F r(\mathcal{C}, \mathcal{D})$ is a welldefined category containing $\operatorname{Fr}(\mathcal{C}, \mathcal{D})$ as full subcategory (here $\operatorname{Fr}(\mathcal{C}, \mathcal{D})$ denotes the category of Frobenius functors). In the same vein of [3], we can define a functor $\oplus: h \mathcal{A} \times h \mathcal{A} \rightarrow h \mathcal{A}(h \mathcal{A}=h \operatorname{Fr}(\mathcal{C}, \mathcal{D})$ and $\mathcal{A}=\operatorname{Fr}(\mathcal{C}, \mathcal{D}))$ which associate to each pair of homological Frobenius functor $F$ and $F^{\prime}$ their direct sum $F \oplus G$. Then we can construct the Grothendieck group $\left(K_{0}(h \mathcal{A}),+\right)$, where $K_{0}(h \mathcal{A})$ is a set of representatives (up to natural isomorphisms) of all homological Frobenius functors from $\mathcal{C}$ to $\mathcal{D}$. We call this group the homological functorial Grothendieck group of $\mathcal{C}$ and $\mathcal{D}$, and denote it by $h F K_{0}(\mathcal{C}, \mathcal{D})$. Note that $F K_{0}(\mathcal{C}, \mathcal{D})$, the functorial Grothendieck group, is a subgroup of $h F K_{0}(\mathcal{C}, \mathcal{D})$.

We now study when homological Frobenius functors preserve Gorenstein categories.

Lemma 3.5. Let $\mathcal{C}$ and $\mathcal{D}$ be two Grothendieck categories and let $(F, G), F: \mathcal{C} \rightarrow \mathcal{D}$, be a homological Frobenius pair. Suppose $F$ is exact and $G$ is faithful. If $\mathcal{C}$ has a generator 
$P$ of finite projective dimension then $F(P)$ is a generator of $\mathcal{D}$ with finite projective dimension.

Proof. $F(P)$ is a generator by Lemma 3.3. But $G$ preserves injective resolutions since $F$ is exact, so we have

$$
\begin{gathered}
\operatorname{Ext}^{n}(F(P), Y)=\operatorname{Hom}_{\mathbf{D}(\mathcal{D})}(F(P), Y[n])=\operatorname{Hom}_{\mathbf{D}(\mathcal{D})}(F(P), \mathbf{i} Y[n])= \\
H^{n}\left(\operatorname{Hom}^{\bullet}(F(P), \mathbf{i} Y)\right)=H^{n}\left(\operatorname{Hom}^{\bullet}(P, G(\mathbf{i} Y))=\operatorname{Hom}_{\mathbf{D}(\mathcal{C})}(P, G(\mathbf{i} Y)[n])=\right. \\
\operatorname{Ext}^{n}(P, G(Y))=0 .
\end{gathered}
$$

THEOREM 3.6. Let $\mathcal{C}$ and $\mathcal{D}$ be two Grothendieck categories and let $(F, G), F: \mathcal{C} \rightarrow$ $\mathcal{D}$, be a homological Frobenius pair. Suppose $F$ is exact and $G$ is faithful. If $\mathcal{C}$ is Gorenstein then $\mathcal{D}$ is Gorenstein.

Proof. Let $P$ be a generator of $\mathcal{C}$ with $p d(P)=n<\infty$ and $E$ an injective cogenerator of $\mathcal{D}$. By Lemma 3.5, it is enough to prove that $i d(F(P))=n<\infty$ and $p d(G(E)) \leq$ $n<\infty$.

Since $F$ is exact, $F=\mathbf{L} F$. Then,

$$
\begin{gathered}
\operatorname{Ext}^{n}(X, F(P)) \cong \operatorname{Hom}_{\mathbf{D}(\mathcal{D})}(X[-n], F(P)) \cong \operatorname{Hom}_{\mathbf{D}(\mathcal{D})}(X[-n], \mathbf{L} F(P)) \cong \\
\operatorname{Hom}_{\mathbf{D}(\mathcal{C})}(\mathbf{R} G(X)[-n], P) \cong \operatorname{Hom}_{\mathbf{K}(\mathcal{C})}(\mathbf{R} G(X)[-n], \mathbf{i} P) .
\end{gathered}
$$

But the complexes $\mathbf{R} G(X)[-n]$ and $\mathbf{i} P$ do not share non-null terms, so indeed $\operatorname{Hom}_{\mathbf{K}(\mathcal{C})}(\mathbf{R} G(X)[-n], \mathbf{i} P)=0$.

Analogously,

$$
\begin{gathered}
\operatorname{Ext}^{n}(F(E), X) \cong \operatorname{Hom}_{\mathbf{D}(\mathcal{D})}(F(E)[-n], X) \cong \\
\operatorname{Hom}_{\mathbf{K}(\mathcal{D})}(F(E)[-n], \mathbf{i} X) \cong \operatorname{Hom}_{\mathbf{K}(\mathcal{D})}(E[-n], G(\mathbf{i}(X)) \cong \\
\operatorname{Hom}_{\mathbf{D}(\mathcal{D})}(E[-n], G(X)) \cong \operatorname{Ext}^{n}(E, G(X))=0
\end{gathered}
$$

(note that $G(\mathbf{i} X)$ is an injective resolution of $X$ since $F$ is exact).

\section{Homological Frobenius complexes.}

Definition 4.1. We say that a complex of $(S, R)$-bimodules $P^{\bullet}$ is a homological Frobenius complex if:

(a) $P^{\bullet}$ is compact in both $\mathbf{D}(M o d-R)$ and $\mathbf{D}(S-M o d)$.

(b) $\operatorname{Hom}_{R}\left(P^{\bullet}, R\right) \cong \operatorname{Hom}_{S}\left(P^{\bullet}, S\right)$ as complexes in both $\mathbf{D}(\operatorname{Mod}-S)$ and $\mathbf{D}(R$ Mod).

Remark. (1) Any tilting complex $T$ of $\left(R, \operatorname{End}_{\mathbf{D}(R)}(T)\right)$-bimodules is a homological Frobenius complex. 
(2) If ${ }_{S} M_{R}$ and ${ }_{S} N_{R}$ are Frobenius bimodules, then the mapping cone of $0: M \rightarrow N$ and $i d_{M}: M \rightarrow M$ are homological Frobenius complexes.

EXAMPLE 4.2. Using [9] we now give a family of examples of homological Frobenius complexes of bimodules that are not tilting.

Let $A$ be a finitely dimensional algebra over a field $K, e \in A$ be an idempotent element such that $\operatorname{Hom}_{K}(A e, K) \cong e A$ as $K$-spaces and $\left\{e_{1}, e_{2}, \ldots, e_{n}\right\}$ be a complete set of primitive orthogonal idempotents of $A$. Take $I=\{1,2, \ldots, n\}$ and set $\Delta=A / A e A$ and $I(e)=\left\{i \in I: e_{i} \Delta=0\right\}$. Then the complex $Q_{0}(e)^{\bullet}$ given by the mapping cone of the multiplication map

$$
\mu_{0}: \oplus_{i \in I(e)} A e_{i} \otimes_{K} e_{i} A \rightarrow A
$$

is a complex of $(A, A)$-bimodules.

By [9, Lemma 5.4], $\operatorname{Hom}_{A}\left(Q_{0}(e)^{\bullet}, A_{A}\right) \cong \operatorname{Hom}_{A}\left(Q_{0}(e),{ }_{A} A\right)$ in $\mathbf{K}\left(\operatorname{Mod}-A^{e}\right)\left(A^{e}=\right.$ $\left.A^{o p} \otimes_{K} A\right)$. Since $Q_{0}(e)^{\bullet}$ is compact as complex of left and right $A$-modules, $Q_{0}(e)^{\bullet}$ is a homological Frobenius $(A, A)$-bimodule which is not a tilting complex of $(A, A)$ bimodules if $\operatorname{dim}(e A e) \neq 2[9$, Remark 4.3].

So, for example if $A=T S_{n}(K)(n \geq 2)$ is the ring of $n \times n$ upper triangular matrices over $K$ and $e=e_{i i}$ is the idempotent matrix with 1 in the $(i, i)$ entry and zero otherwise, then it is easy to see that $\operatorname{Hom}_{K}(e A, K) \cong A e, \operatorname{dim}_{K}(e A e)=1, I(e)=\left\{e_{i i}\right\}$. Hence, we have an example of the above construction.

REMARK 4.3. The definition of homological Frobenius complexes of bimodules may be reformulated in the setting of graded rings and graded modules. Thus, if $A=K \oplus$ $A_{1} \oplus A_{2} \oplus \cdots$ is a connected $\mathbb{N}$-graded $K$-algebra, then $K[-d]$ (the $-d$ 's shift complex of $K$ as a complex concentrated in degree zero) is a graded homological Frobenius complex of $(A, K)$-bimodules (this means that the homological Frobenius property is verified up to some graded shift, that is, there is an isomorphism in the derived categories $\operatorname{Hom}_{A}(K[-d], A) \cong \operatorname{Hom}_{K}(K(l), K)$ for some integer l) with $\mathrm{pd}_{A} K=d$ if and only if $A$ is an $A S$-regular graded algebra of global dimension $d$ and Gorenstein parameter $l$ (see [13, Definition 1.1]).

We will need the following lemmas.

LeMma 4.4. Let $P^{\bullet}$ be a complex of $(S, R)$-bimodules, suppose $P_{R}^{\bullet}$ is perfect and call $\bar{Q}^{\bullet}=\operatorname{Hom}_{R}\left(P^{\bullet}, R\right)$. Then, there is a natural isomorphism of functors in the derived category $\mathbf{D}(R), \operatorname{Hom}_{R}^{\bullet}\left(P^{\bullet},-\right) \cong-\otimes_{R}^{\bullet} \bar{Q}^{\bullet}$.

Proof. Follows by [14, Lemma 17.12] or [5, p. 6], where the proof is done at the level of complexes.

LEMMA 4.5. [12, Proposition 2.9] Let $\mathcal{C}$ be a compactly generated triangular category. The following assertions are equivalent for a coproduct preserving cohomological functor $F: \mathcal{C} \rightarrow \mathbf{A b}:$

(i) $F$ preserves products.

(ii) $F$ preserves products of compact objects.

(iii) There is a compact object $C$ in $\mathcal{C}$ such that $F \cong \operatorname{Hom}_{\mathcal{C}}(C,-)$.

TheORem 4.6. Let $P^{\bullet}$ be a complex of $(S, R)$-bimodules, $Q^{\bullet}=\mathbf{R} \operatorname{Hom}_{S}\left(P^{\bullet}, S\right)$ and $\bar{Q}^{\bullet}=\mathbf{R} \operatorname{Hom}_{R}\left(P^{\bullet}, R\right)$. The following conditions are equivalent.

(i) $P^{\bullet}$ is a homological Frobenius complex. 
(ii) $\left(P^{\bullet} \otimes_{R}^{\mathbf{L}}-, \mathbf{R} \operatorname{Hom}_{S}\left(P^{\bullet},-\right)\right)$ is a Frobenius pair.

(iii) $\left(-\otimes_{S}^{\mathbf{L}} P^{\bullet}, \mathbf{R} \operatorname{Hom}_{R}\left(P^{\bullet},-\right)\right)$ is a Frobenius pair.

(iv) ${ }_{S} P^{\bullet}$ and $P_{R}^{\bullet}$ are compact and there is a natural isomorphism of functors $\mathbf{R} \operatorname{Hom}_{R}\left(Q^{\bullet},-\right) \cong P^{\bullet} \otimes_{R}^{\mathbf{L}}-$.

(v) ${ }_{S} P^{\bullet}$ and $P_{R}^{\bullet}$ are compact and there is a natural isomorphism of functors $\mathbf{R} \operatorname{Hom}_{S}(\bar{Q},-) \cong-\otimes P_{S}^{\mathbf{L}} P^{\bullet}$.

Proof. (i) $\Rightarrow$ (ii) We have two adjoint pairs of functors $\left(P^{\bullet} \otimes_{R}^{\bullet}-, \operatorname{Hom}_{S}^{\bullet}\left(P^{\bullet},-\right)\right)$ and $\left(Q^{\bullet} \otimes_{S}^{\bullet}-, \operatorname{Hom}_{R}^{\bullet}\left(Q^{\bullet},-\right)\right)$, and by Lemma 4.4 we know that $\operatorname{Hom}_{S}^{\bullet}\left(P^{\bullet},-\right) \cong Q^{\bullet} \otimes_{S}^{\bullet}-$, so we just have to see that $P^{\bullet} \otimes_{R}^{\bullet}-\cong \operatorname{Hom}_{R}^{\bullet}\left(Q^{\bullet},-\right)$.

But $\operatorname{Hom}_{R}\left(Q^{\bullet}, R\right) \cong \operatorname{Hom}_{R}\left(\operatorname{Hom}_{R}\left(P^{\bullet}, R\right), R\right) \cong P^{\bullet} \otimes_{R} R$, so by Lemma 4.4 we get that $P^{\bullet} \otimes_{R}^{\bullet}-\cong \operatorname{Hom}_{R}^{\bullet}\left(Q^{\bullet},-\right)$.

(ii) $\Rightarrow$ (i) By Lemma $4.5_{S} P^{\bullet}$ and $P_{R}^{\bullet}$ are compact, so $Q_{S}^{\bullet}$ and ${ }_{R} Q^{\bullet}$ are also compact. Hence, $P^{\bullet} \otimes_{R}^{\bullet} \cong \operatorname{Hom}_{R}^{\bullet}\left(Q^{\bullet},-\right)$ by Lemma 4.4 and then ${ }_{S} P_{R}^{\bullet} \cong{ }_{S} \operatorname{Hom}_{R}\left(Q^{\bullet}, R\right)_{R}$, therefore

$$
\operatorname{Hom}_{R}\left(P^{\bullet}, R\right) \cong \operatorname{Hom}_{R}\left(\operatorname{Hom}_{R}\left(Q^{\bullet}, R\right), R\right) \cong Q^{\bullet}=\operatorname{Hom}_{S}\left(P^{\bullet}, S\right)
$$

and we are done.

(i) $\Rightarrow$ (iii) and (iii) $\Rightarrow$ (i) have similar proofs to (i) $\Rightarrow$ (ii) and (ii) $\Rightarrow$ (i) respectively.

(ii) $\Leftrightarrow$ (iv) and (iii) $\Leftrightarrow$ (v) are straightforward.

Definition 4.7. An extension of DG-algebras $A \mid B$ is called derived Frobenius if $A_{B}$ is finitely generated and projective and $A \cong \operatorname{Hom}_{B}^{\bullet}(A, B)$ in the derived categories of left $A$-DG-modules and right $B$-DG-modules.

The following result is a derived analogue to the endomorphism ring theorem for Frobenius extensions [11, Theorem 2.1].

Proposition 4.8. Let ${ }_{S} P_{R}^{\bullet}$ be a homological Frobenius complex and call $E=$ $\operatorname{Hom}_{S}^{\bullet}\left(P^{\bullet}, P^{\bullet}\right)$ its endomorphism DG-algebra. Then the DG-algebras extension $E \mid R$ is derived Frobenius.

Proof. For all $i \in \mathbb{Z}$ we have

$$
\begin{gathered}
H^{i}(E)=H^{i}\left(\operatorname{Hom}_{S}^{\bullet}\left(P^{\bullet}, P^{\bullet}\right)\right)=\operatorname{Hom}_{\mathbf{D}(S)}\left(P^{\bullet}, P^{\bullet}[i]\right) \\
\cong \operatorname{Hom}_{\mathbf{D}(S)}\left(P^{\bullet}, P^{\bullet}[i] \otimes_{R} R\right) \cong \operatorname{Hom}_{\mathbf{D}(R)}\left(\operatorname{Hom}_{S}^{\bullet}\left(P^{\bullet}, P^{\bullet}\right), R[i]\right) \\
\cong \operatorname{Hom}_{\mathbf{D}(R)}(E, R[i]) \cong H^{i}\left(\operatorname{Hom}_{R}(E, R)\right) .
\end{gathered}
$$

Therefore, $E \cong \operatorname{Hom}_{R}(E, R)$ in the derived categories.

Note that by symmetry in the above proposition if $E^{\prime}=\operatorname{Hom}_{R}^{\bullet}\left(P^{\bullet}, P^{\bullet}\right)$, then $E^{\prime} \mid S$ is derived Frobenius.

Now we give a converse of the above result. The following proposition is the derived version of [11, Theorem 2.2].

Proposition 4.9. Let ${ }_{S} P_{R}^{\bullet}$ be a complex of $(S, R)$-bimodules such that both ${ }_{S} P^{\bullet}$ and $P_{R}^{\bullet}$ are compact, call $E=\operatorname{Hom}_{S}^{\bullet}\left(P^{\bullet}, P^{\bullet}\right)$ and suppose that $E \mid R$ is a derived Frobenius 
extension and that

$$
\operatorname{RHom}_{E}^{\bullet}\left(P^{\bullet}, E\right)_{S} \cong{ }_{R} \operatorname{Hom}_{S}\left(P^{\bullet}, S\right)_{S}
$$

in the derived categories.

Then ${ }_{S} P_{R}^{\bullet}$ is a homological Frobenius complex.

Proof. It follows by the following isomorphism chain in the derived categories:

$$
\begin{aligned}
\operatorname{Hom}_{R}\left(P^{\bullet}, R\right) & \cong \operatorname{Hom}_{R}\left(E \otimes_{E}^{\bullet} P^{\bullet}, R\right) \cong \operatorname{Hom}_{E}^{\bullet}\left(P^{\bullet}, \operatorname{Hom}_{R}^{\bullet}(E, R)\right) \cong \\
& \cong \operatorname{Hom}_{E}^{\bullet}\left(P^{\bullet}, E\right) \cong \operatorname{Hom}_{S}\left(P^{\bullet}, S\right) .
\end{aligned}
$$

Corollary 4.10. Let ${ }_{S} P_{R}^{\bullet}$ be a two-sided tilting complex, $E=\operatorname{Hom}_{S}^{\bullet}\left(P^{\bullet}, P^{\bullet}\right)$ and $E^{\prime}=\operatorname{Hom}_{R}^{\bullet}\left(P^{\bullet}, P^{\bullet}\right)$. Then $E \mid R$ and $E^{\prime} \mid S$ are Frobenius extensions of rings.

Proof. Now we have $E \cong H^{0}(E)$ and $E^{\prime} \cong H^{0}\left(E^{\prime}\right)$, so the derived Frobenius extensions $E \mid R$ and $E^{\prime} \mid S$ are usual ring extensions, and hence they are Frobenius extension of rings.

5. Homological Frobenius bimodules. In this section, we consider a particular case of stalk complexes, i.e. bimodules.

Definition 5.1. Let $T$ be an $(S, R)$-bimodule. We say that $T$ is a homological Frobenius bimodule if:

(a) ${ }_{S} T$ and $T_{R}$ have finite projective resolutions of finitely generated projective modules.

(b) There are isomorphisms of $(R, S)$-bimodules $\operatorname{Hom}_{S}(T, S) \cong \operatorname{Hom}_{R}(T, R)$, $\operatorname{Ext}_{R}^{i}(T, R) \cong \operatorname{Ext}_{S}^{i}(T, S)$ for all $i \geq 1$.

Remarks. (1) Any tilting bimodule ${ }_{S} T_{R}\left(S=\operatorname{End}_{R}(T)\right)$ is a homological Frobenius bimodule.

(2) Any $R$-module of finite projective dimension over a commutative ring is trivially a homological Frobenius $(R, R)$-bimodule, so we see there are homological Frobenius bimodules that are not tilting.

(3) If $M$ is an $(S, R)$-bimodule, then it is an exercise to check that the following assertions are equivalent:

(i) $M$ is a homological Frobenius $(S, R)$-bimodule.

(ii) $\cdots 0 \rightarrow M \rightarrow 0 \rightarrow \cdots$ is a homological Frobenius complex.

EXAMPLE 5.2. (1) Let $R$ be a ring and $G$ be a finite group. Then $R$ is a homological Frobenius $(R, R[G]$ )-bimodule (the structure of right $R[G]$-module is given by the trivial action) if and only if the cohomological dimension of $G$ is zero (that is $|G|$ is invertible in $R$ ).

(2) Homological Frobenius bimodules do not preserve in general Gorenstein properties: Let $R=S \oplus T$, where $S$ is a Gorenstein ring and $T$ is not a Gorenstein ring. Then $R$ is not a Gorenstein ring but $S$ is a Frobenius (so homological Frobenius) $(S, R)$-bimodule.

The following result will be useful. 
LEMMA 5.3. Let $M_{R}$ be a right $R$-module with a finite projective resolution of finitely generated right $R$-modules and $\operatorname{Ext}_{R}^{i}(M, R)=0$ for all $i \geq 1$. Then $M_{R}$ is projective.

Proof. Let

$$
0 \rightarrow P^{n} \rightarrow P^{n-1} \rightarrow \cdots \rightarrow P^{0} \rightarrow M \rightarrow 0
$$

be a projective resolution with all $P^{i}$ finitely generated. Then

$$
0 \rightarrow \operatorname{Hom}_{R}(M, R) \rightarrow \operatorname{Hom}_{R}\left(P^{0}, R\right) \rightarrow \cdots \rightarrow \operatorname{Hom}_{R}\left(P^{n}, R\right) \rightarrow 0
$$

splits since it is exact and $\operatorname{Hom}_{R}\left(P^{i}, R\right)$ are all projective left $R$-modules. Hence, applying again $\operatorname{Hom}_{R}(-, R)$ we get that the original sequence splits since the $P^{i}$ are all reflexive, so $M_{R}$ is projective.

As an application of above lemma we get the following result.

Proposition 5.4. Let $A$ and $B$ be two rings, $M$ an $(A, B)$-bimodule, and suppose $B$ satisfies the following condition: If $B \cong B \oplus X(X \in B$-Mod) then $X=0$. Then if we let $R=\left(\begin{array}{cc}A & M \\ 0 & B\end{array}\right)$ and $e=\left(\begin{array}{ll}0 & 0 \\ 0 & 1\end{array}\right) \in R$, the following assertions are equivalent.

(i) Re is an $(R, B)$-homological Frobenius bimodule.

(ii) $M=0$.

(iii) Re is an $(R, B)$-Frobenius bimodule.

Proof. (i) $\Rightarrow$ (ii) Note that $B \cong e$ Re as rings and $\operatorname{Hom}_{R}(R e, R) \cong e R \cong B$ as $(B, R)$ bimodules. On the other hand, $\operatorname{Hom}_{B}(\operatorname{Re}, B) \cong \operatorname{Hom}_{B}(M \oplus B, B) \cong \operatorname{Hom}_{B}(M, B) \oplus B$ as $(B, R)$-bimodules. Hence, we obtain $\operatorname{Hom}_{B}(M, B)=0$.

Since ${ }_{R} R e$ is projective, we have $\operatorname{Ext}_{B}^{i}(\operatorname{Re}, B) \cong \operatorname{Ext}_{R}^{i}(\operatorname{Re}, R)=0$ for all $i \geq 1$. Therefore, $\operatorname{Ext}_{B}^{i}(M, B)=0$ for all $i \geq 1$.

Now $R e_{B}$ has a finite projective resolution of finitely generated modules, so $M_{B}$ does too. Hence, the result follows from Lemma 5.3 since $\operatorname{Hom}_{B}(M, B)=0$.

(ii) $\Rightarrow($ iii $)$ and $($ iii $) \Rightarrow($ i $)$ are clear.

In the above proposition we can find conditions for $R e$ not to be a tilting $R$-module when $R e$ is homological Frobenius. The only condition to check for $R e$ to be tilting is the existence of an exact sequence

$$
0 \rightarrow R \rightarrow L^{1} \rightarrow \cdots \rightarrow L^{m} \rightarrow 0
$$

with $L^{i} \in \operatorname{add}(R e)$. If this sequence exists, it is easy to find a monomorphism $A \rightarrow L$ with $L \in \operatorname{add}\left({ }_{A} M\right)$.

Hence, if we do not want $R e$ to be tilting, we just have to find an $A$ not isomorphic to any submodule of $L \in \operatorname{add}\left({ }_{A} M\right)$.

LemMA 5.5. If $M \otimes_{R}^{\mathbf{L}}-$ is Frobenius then $M_{R}$ is quasi-isomorphic to a perfect complex of right $R$-modules.

Proof. By Lemma $4.5 H^{0}\left(M \otimes_{R}^{\mathbf{L}}-\right) \cong \operatorname{Hom}_{\mathbf{D}(R-M o d)}(C,-)$ for some compact object $C$ in $\mathbf{D}(R-M o d)$, hence $C$ is quasi-isomorphic to a perfect complex $Q^{\bullet}$. But then

$$
\left.\operatorname{Tor}_{i}^{R}(M, R) \cong H^{0}\left(M \otimes_{R}^{\mathbf{L}} R[i]\right)\right) \cong \operatorname{Hom}_{\mathbf{K}(R)}\left(Q^{\bullet}, R[i]\right) \cong H^{i}\left(H_{R}\left(Q^{\bullet}, R\right)\right),
$$

so $H^{i}\left(\operatorname{Hom}_{R}\left(Q^{\bullet}, R\right)\right)=0$ for $i \neq 0$ and $M \cong H^{0}\left(\operatorname{Hom}_{R}\left(Q^{\bullet}, R\right)\right)$. 
Therefore, $M$ is quasi-isomorphic to the perfect complex of right $R$-modules $\operatorname{Hom}_{R}\left(Q^{\bullet}, R\right)$.

An interesting fact about homological Frobenius functors between module categories is that, having left and right adjoints, they are nothing but Frobenius functors.

Proposition 5.6. Let $(F, G),(G, H)$ be adjoint pairs, where $F: R$-Mod $\rightarrow S$-Mod, and suppose $\mathbf{L} F \cong \mathbf{R} H$ in the derived categories. Then $F \cong H$, that is, the adjoint pair $(F, G)$ is Frobenius.

Proof. By the Eilenberg-Watts theorem there is a bimodule ${ }_{S} M_{R}$ such that $F \cong M \otimes_{R}-, G \cong \operatorname{Hom}_{S}(M,-)$ and $H \cong \operatorname{Hom}_{R}\left(\operatorname{Hom}_{S}(M, S),-\right)$. Since $G$ is exact, ${ }_{S} M$ is projective, and since $G$ is a left adjoint, it preserves direct sums. Hence, ${ }_{S} M$ is a finitely generated projective. But then $S^{m}=M \oplus M^{\prime}$, so $S^{m} \cong \operatorname{Hom}_{S}\left(S^{m}, S\right) \cong$ $\operatorname{Hom}_{S}(M, S) \oplus \operatorname{Hom}_{S}\left(M^{\prime}, S\right)$ and then $\operatorname{Hom}_{S}(M, S)$ is a finitely generated projective right $S$-module.

Now $\mathbf{L} F \cong \mathbf{R} H$, so $\operatorname{Hom}_{S}(M, S)$ is isomorphic to a perfect complex in $\mathbf{D}(R)$ and then $\operatorname{Hom}_{S}(M, S)$ has a finite projective resolution of finitely generated left $R$-modules.

But $\quad M \cong M \otimes_{R}^{\mathrm{L}} R \cong \operatorname{Hom}_{R}\left(\operatorname{Hom}_{S}(M, S), I_{R}^{\bullet}\right) \quad$ in $\mathbf{D}(S), \quad$ so we have ${ }_{S} M \cong{ }_{S} \operatorname{Hom}_{R}\left(\operatorname{Hom}_{S}(M, S), R\right)$ in $S$-Mod (and in Mod-R naturally) and $\operatorname{Hom}_{R}\left(\operatorname{Hom}_{S}(M, S), I_{R}^{\bullet}\right)$ is an injective resolution of $M$ in $S$-Mod (note that $\operatorname{Hom}_{S}(M, S)_{S}$ is projective).

Therefore, $\operatorname{Ext}_{R}^{i}\left(\operatorname{Hom}_{S}(M, S), R\right)=0 \forall i>0$ and then ${ }_{R} \operatorname{Hom}_{S}(M, S)$ is finitely generated and projective (Lemma 5.3), so we have $R^{n} \cong \operatorname{Hom}_{S}(M, S) \oplus L$. But then $R^{n} \cong \operatorname{Hom}_{R}\left(R^{n}, R\right) \cong \operatorname{Hom}_{R}\left(\operatorname{Hom}_{S}(M, S), R\right) \cong M$ in Mod-R, so $M_{R}$ is finitely generated and projective.

We have just proved that $(F, G)$ is indeed a Frobenius pair.

We now prove that homological Frobenius adjoint pairs of functors between module categories are always represented by homological Frobenius bimodules.

Corollary 5.7. Let $(F, G)$ be an adjoint pair of functors, $F: R$-Mod $\rightarrow S$-Mod. The following assertions are equivalent:

(i) $(F, G)$ is a homological Frobenius adjoint pair.

(ii) There is a homological Frobenius bimodule ${ }_{S} M_{R}$ such that $F \cong M \otimes_{R}-$ and $G \cong \operatorname{Hom}_{S}(M,-)$.

Proof. (i) $\Rightarrow$ (ii) By the Eilenberg-Watts theorem there is a bimodule ${ }_{S} M_{R}$ such that $F \cong M \otimes_{R}-$ and $G \cong \operatorname{Hom}_{S}(M,-)$. Since $\left(M \otimes_{R}^{\mathbf{L}}-, \mathbf{R} \operatorname{Hom}_{S}(M,-)\right)$ and $\left(-\otimes_{S}^{\mathbf{L}}\right.$ $\left.M, \mathbf{R} \operatorname{Hom}_{R}(M,-)\right)$ are Frobenius pairs (Theorem 4.6), it follows that $M_{R}$ and ${ }_{S} M$ are compact in the respective derived categories, so they are quasi-isomorphic to perfect complexes (Lemma 5.5).

Now by Theorem 4.6 we have that $\mathbf{R} \operatorname{Hom}_{R}(M, R) \cong \mathbf{R} \operatorname{Hom}_{S}(M, S)$ in the derived categories, so taking homology, $\operatorname{Ext}_{R}^{i}(M, R) \cong \operatorname{Ext}_{S}^{i}(M, S)$ for all $i \geq 0$ as $R-S$ bimodules.

Therefore, we get that ${ }_{S} M_{R}$ is a homological Frobenius bimodule.

(ii) $\Rightarrow$ (i) Follows from Theorem 4.6.

Proposition 5.8. Let $M$ be a homological Frobenius $(S, R)$-bimodule. Then:

(a) ${ }_{S} M$ has a finite projective resolution of finitely generated left $S$-modules.

(b) $M_{R}$ has a finite projective resolution of finitely generated right $R$-modules. 
Proof. (a) ${ }_{S} M$ is quasi-isomorphic to a perfect complex in $\mathbf{D}(S$-Mod) since $\mathbf{R} \operatorname{Hom}_{S}(M,-)$ (and so $\left.\operatorname{Hom}_{\mathbf{D}(S-M o d)}(M,-)\right)$ preserves coproducts.

(b) Follows by Lemma 5.5.

Lemma 5.9. Let $M$ be a homological Frobenius $(S, R)$-bimodule. Then:

(a) $\operatorname{Hom}_{R}\left(\operatorname{Hom}_{S}(M, E), R\right) \cong \operatorname{Hom}_{S}(E, M)$ and $\operatorname{Ext}_{R}^{i}\left(\operatorname{Hom}_{S}(M, E), R\right) \cong$ $\operatorname{Ext}_{S}^{i}(E, M)$ for every injective $E \in S$-Mod.

(b) $\operatorname{Hom}_{R}\left(\operatorname{Hom}_{S}(M, S), E^{\prime}\right) \cong M \otimes_{R} E^{\prime} \quad$ and $\quad \operatorname{Hom}_{R}\left(\operatorname{Ext}_{S}^{i}(M, S), E^{\prime}\right) \cong$ $\operatorname{Tor}_{i}^{R}\left(M, E^{\prime}\right)$ for every injective $E^{\prime} \in R$-Mod.

Proof. Let $E$ be an injective left $R$-module. Then, from the natural isomorphism,

$$
\operatorname{Hom}_{\mathbf{D}(R)}\left(\mathbf{R H o m}{ }_{S}(M, E), R[i]\right) \cong \operatorname{Hom}_{\mathbf{D}(S)}\left(E, M \otimes_{R}^{\mathbf{L}} R[i]\right)
$$

we get (a).

To prove (b), we just have to use

$$
\operatorname{Hom}_{\mathbf{D}(R)}\left(\mathbf{R} \operatorname{Hom}_{S}(M, S), E^{\prime}[i]\right) \cong \operatorname{Hom}_{\mathbf{D}(S)}\left(S, M \otimes_{R}^{\mathbf{L}} E^{\prime}[i]\right) .
$$

Using the same arguments we get the following.

Corollary 5.10. Let $M$ be a homological Frobenius $(S, R)$-bimodule. Then:

(a) If $S$ is injective, then $\operatorname{Ext}_{R}^{i}\left(\operatorname{Hom}_{S}(M, S), R\right)=0$ for $i \neq 0$ and

$$
\operatorname{Hom}_{R}\left(\operatorname{Hom}_{S}(M, S), R\right) \cong M .
$$

(b) If ${ }_{R} R$ is injective, then $\operatorname{Hom}_{R}\left(\operatorname{Ext}_{S}^{i}(M, M), R\right) \cong \operatorname{Ext}_{S}^{i}(M, M), \forall i \geq 0$.

Proposition 5.11. Let $M$ be a homological Frobenius $(S, R)$-bimodule. If $M_{R}$ is projective then ${ }_{S} M$ is projective, and hence ${ }_{S} M_{R}$ is a classical Frobenius bimodule.

Proof. ${ }_{S} M$ is projective by (b) in Lemma 5.9 choosing ${ }_{R} E^{\prime}$ an injective cogenerator.

Now, given $X \in S$-Mod, $Y \in R$-Mod, $I_{Y}^{\bullet}$ an injective resolution of $Y$ and $P_{X}^{\bullet}$ a projective resolution of $X$, we have

$$
\begin{aligned}
& \operatorname{Hom}_{R}\left(\operatorname{Hom}_{S}(M, X), Y\right) \cong H^{0}\left(\operatorname{Hom}_{R}\left(\operatorname{Hom}_{S}(M, X), I_{Y}^{\bullet}\right)\right) \\
& \cong \operatorname{Hom}_{\mathbf{D}(R)}\left(\mathbf{R} \operatorname{Hom}_{S}(M, X), Y\right) \cong \operatorname{Hom}_{\mathbf{D}(S)}\left(X, M \otimes_{R}^{\mathbf{L}} Y\right) \cong \\
& \cong H^{0}\left(\operatorname{Hom}_{S}\left(P_{X}^{\bullet}, M \otimes_{R} Y\right)\right) \cong \operatorname{Hom}_{S}\left(X, M \otimes_{S} Y\right) .
\end{aligned}
$$

Hence, ${ }_{S} M_{R}$ is a Frobenius bimodule.

Proposition 5.12. Let $M$ be a homological Frobenius $(S, R)$-bimodule. Suppose that $R$ is Iwanaga Gorenstein.

(a) If ${ }_{S} M$ is projective then $\operatorname{Hom}_{S}(M, S)$ is a Gorenstein projective left $R$-module and $M_{R}$ is projective. In particular, ${ }_{S} M_{R}$ is a (classical) Frobenius bimodule.

(b) If ${ }_{S} M$ is injective then $\operatorname{Hom}_{S}(M, E)$ is a Gorenstein projective left R-module for all ${ }_{S} E$ injective. 
Proof. (a) Let $N \in R$-Mod with $\operatorname{inj} \operatorname{dim}(N)<\infty$. By (b) in Lemma 5.9 applied to a finite injective resolution of ${ }_{R} N$ we get that $M \otimes_{R} N \cong \operatorname{Hom}_{R}\left(\operatorname{Hom}_{S}(M, S), N\right)$ and $\operatorname{Ext}_{R}^{i}\left(\operatorname{Hom}_{S}(M, S), N\right)=0 \forall i>0$. Hence, since $R$ is Gorenstein, we have that $\operatorname{Hom}_{S}(M, S)$ is Gorenstein projective. On the other hand, again by (b) of Lemma 5.9, $\operatorname{Tor}_{i}^{R}\left(M, E^{\prime}\right)=0$ for all ${ }_{R} E^{\prime}$ injective, so $M_{R}$ is Gorenstein flat. But $M_{R}$ has finite projective dimension and $M_{R}$ is finitely presented, therefore $M_{R}$ is projective.

(b) We have $\operatorname{Ext}_{S}^{i}(E, M)=0$ for all ${ }_{S} E$ injective and all $i>0$. Then, by (a) in Lemma 5.9, Ext $t_{R}^{i}\left(\operatorname{Hom}_{S}(M, E), R\right)=0$ and then $\operatorname{Hom}_{S}(M, E)$ is Gorenstein projective as a left $R$-module.

Corollary 5.13. Let $M$ be a homological Frobenius $(S, R)$-bimodule. If $R_{R}$ and ${ }_{S} S$ are injective then ${ }_{S} M_{R}$ is a classical Frobenius bimodule.

Proof. Follows by Proposition 5.11.

Let $f: R \rightarrow S$ be a ring homomorphism and $f_{*}: S$-Mod $\rightarrow R$-Mod the usual restriction of scalars functor. The induced functor between the derived categories, $\mathbf{D}\left(f_{*}\right): \mathbf{D}(S) \rightarrow \mathbf{D}(R)$ is right adjoint to $S \otimes_{R}^{\mathbf{L}}-$ and left adjoint to $\mathbf{R} \mathrm{Hom}_{R}(S,-)$.

Definition 5.14. The ring homomorphism $f: R \rightarrow S$ is said to be homological Frobenius if the functor $f_{*}$ is homological Frobenius.

It is possible to characterize when the restriction of scalars functor $f_{*}: S$ $M o d \rightarrow R-M o d$ is homological Frobenius. That is exactly the case when the ring homomorphism $f$ is Frobenius in the usual sense. This result generalizes Propositions 1.5 and 1.7 in [1].

Proposition 5.15. Let $f: R \rightarrow S$ be a ring homomorphism. The following assertions are equivalent:

(i) The functor $f_{*}: S-M o d \rightarrow R-$ Mod is homological Frobenius.

(ii) (a) ${ }_{R} S$ has a finite projective resolution of finitely generated left $R$-modules.

(b) ${ }_{S} S$ has an injective resolution of the form $\operatorname{Hom}_{R}\left(S,{ }_{R} I^{\bullet}\right)$, where ${ }_{R} I^{\bullet}$ is an injective resolution of $R$ as a left $R$-module, and there is a quasi-isomorphism of complexes of $(S, R)$-bimodules ${ }_{S} S_{R} \cong{ }_{S} \operatorname{Hom}_{R}\left(S,{ }_{R} I^{\bullet}\right)_{R}$. In particular, ${ }_{S} S_{R} \cong{ }_{S} \operatorname{Hom}_{R}(S, R)_{R}$ as $(S, R)$-bimodules.

(iii) $f: R \rightarrow S$ is a Frobenius homomorphism.

Proof. (i) $\Rightarrow$ (ii) By hypothesis $S \otimes \mathbf{L}-$ and $\mathbf{R} \operatorname{Hom}_{R}(S,-)$ are triangulated equivalent as functors from $\mathbf{D}(R)$ to $\mathbf{D}(S)$ via $\phi: S \otimes^{\mathbf{L}}-\Rightarrow \mathbf{R} \operatorname{Hom}_{R}(S,-)$.

Note that $\operatorname{Hom}_{\mathbf{D}(R)}(S, X)=H^{n}(\mathbf{R} H \operatorname{Hom}(S, \mathbf{i} X[-n]))$, so if $\mathbf{R} H_{o m}(S,-)$ preserves arbitrary coproducts, so does $\operatorname{Hom}_{\mathbf{D}(R)}(S,-)$. Thus, ${ }_{R} S$ is a compact object in $\mathbf{D}(R)$ and therefore ${ }_{R} S$ is isomorphic to a perfect complex in $\mathbf{D}(R),\left(P^{\bullet}, \delta^{\bullet}\right)$ :

$$
\cdots \rightarrow 0 \rightarrow 0 \rightarrow P^{n} \stackrel{\delta^{n}}{\rightarrow} P^{n+1} \rightarrow \cdots \rightarrow P^{i} \rightarrow \cdots \rightarrow P^{m} \rightarrow 0 \rightarrow 0 \rightarrow \cdots
$$

We can suppose that $S \cong H^{i}\left(P^{\bullet}\right)$ and $H^{j}\left(P^{\bullet}\right)=0$ for $j \neq 0$. Then,

$$
0 \rightarrow P^{n} \rightarrow P^{n+1} \rightarrow \cdots \rightarrow P^{i-1} \rightarrow \operatorname{Ker}^{i} \rightarrow S \rightarrow 0
$$

(note that $\operatorname{Ker} \delta^{i} \oplus \operatorname{Im} \delta^{i} \cong P^{i}$ ) is a finite projective resolution of ${ }_{R} S$ and so ${ }_{R} S$ satisfies (a). 
Let $R \rightarrow{ }_{R} I^{\bullet}$ be an injective resolution of $R$ in $\mathbf{D}(R)$, then we have the following natural isomorphisms in $\mathbf{D}(S)$ :

$$
S \cong S \otimes_{R}^{\bullet} R=S \otimes_{R}^{\mathbf{L}} R \cong \mathbf{R} \operatorname{Hom}_{R}(S, R)=\operatorname{Hom}_{R}\left(S,{ }_{R} I^{\bullet}\right)
$$

Therefore, $\quad 0=H^{i}(S) \cong H^{i}\left(\operatorname{Hom}_{R}\left(S, I_{R}^{\bullet}\right)\right) \quad \forall i \neq 0 \quad$ and $\quad S=H^{0}(S) \cong$ $H^{0}\left(\operatorname{Hom}_{R}\left(S,{ }_{R} I^{\bullet}\right)\right)=\operatorname{Hom}_{R}(S, R)$ as left $S$-modules.

Hence, $\operatorname{Hom}_{R}\left(S,{ }_{R} I^{\bullet}\right)$ is an injective resolution of ${ }_{S} S$. On the other hand, for any $r \in R$, the left $R$-module map $\lambda_{r}: R \rightarrow R, x \mapsto x r$ induces a commutative diagram

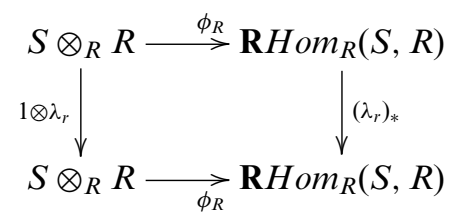

Thus, $\phi_{R} \circ\left(1 \otimes \lambda_{r}\right)(s \otimes 1)=\left(\lambda_{r}\right)_{*} \circ \phi_{R}(s \otimes 1)$ for all $s \in S$ and then $\phi_{R}(s \otimes r)=$ $\phi_{R}(s \otimes 1) r$. Thus, we have the quasi-isomorphism of complexes of $(S, R)$-bimodules ${ }_{S} S_{R} \cong{ }_{S} \operatorname{Hom}_{R}\left(S,{ }_{R} I^{\bullet}\right)_{R}$.

(ii) $\Rightarrow$ (iii) By (b), $\operatorname{Ext}_{R}^{i}(S, R)=0$ for all $i \geq 1$. This, together with (a), gives us that $S_{R}$ is a finitely generated projective module by Lemma 5.3 and then the result follows.

$($ iii $) \Rightarrow(i) \mathrm{I}$ is clear.

Recall [4] that a ring extension $R \subseteq S$ is said to be totally reflexive if:

(a) there is an isomorphism $\operatorname{Hom}_{R}(S, R) \cong S$ of $(S, R)$-bimodules.

(b) $S_{R}$ is a totally reflexive module (that is, it has a complete projective resolution of finitely generated left $R$-modules. If $R$ is left noetherian and ${ }_{R} S$ is finitely generated, then it is totally reflexive if and only if it is a Gorenstein projective module).

Proposition 5.16. Let $R \subseteq S$ be a ring extension. The following assertions are equivalents.

(i) $R \subseteq S$ is totally reflexive and $S_{R}$ has a finite projective resolution of finitely generated modules.

(ii) $R \subseteq S$ is a Frobenius extension.

Proof. Follows by Lemma 5.3.

Proposition 5.17. Let $R \subseteq S$ be a ring extension. Suppose $R$ has finite global dimension. The following assertions are equivalent.

(i) $R \subseteq S$ is totally reflexive.

(ii) $R \subseteq S$ is a Frobenius extension.

Proof. Straightforward.

The following result generalizes [7, Theorem 3.5], where it was proved for extensions of group rings $R \subseteq R[G]$.

COROLlARY 5.18. (Gorenstein ascent theorem) Let $f: R \rightarrow S$ be a Frobenius ring homomorphism. If $R$ is an Iwanaga Gorenstein ring then $S$ is an Iwanaga Gorenstein ring.

Proof. Follows by (ii) in Proposition 5.15. 
ACKNOWLEDGEMENTS. The authors were partially supported by the grant MTM2011-27090 from Ministerio de Ciencia e Innovación, and Proyecto de Excelencia de la Junta de Andalucía P07-FQM-03128, which in part had funds from Fondos Feder.

\section{REFERENCES}

1. H. Abe and M. Hoshino, Frobenius extensions and tilting complexes, Alg. Rep. Theory 11 (2008), 215-232.

2. S. Caenepeel, G. Militaru and S. Zhu, Frobenius and seperable functors for generalized module categories and nonlinear equations, Lecture Notes in Mathematics, vol. 1787 (Springer, Berlin, Germany, 2002).

3. F. Castaño Iglesias, J. Gómez Torrecillas and C. Nastasescu, Frobenius functors: Applications, Comm. Algebra 27(10) (1999), 4879-4900.

4. X.-W. Chen, Totally reflexive extensions and modules, J. Algebra 379(1) (2013) 322-332.

5. H. Chen and C. Xi, Homological ring epimorphisms and recollements from exact pairs,

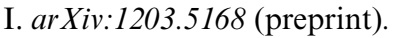

6. E. Enochs, S. Estrada and J. R. García Rozas, Gorenstein categories and Tate cohomology on projective schemes, Math. Nachr. 281(4) (2008), 525-540.

7. E. Enochs, I. Herzog and S. Park, Cyclic quiver rings and polycyclic-by-finite group rings, Houston J. Math. 25(1) (1999), 113.

8. E. Enochs and O. Jenda, Relative homological algebra, vol. 2, De Gruyter Expositions in Mathematics, vol. 54 (De Gruyter, Berlin, Germany, 2011).

9. M. Hoshino and Y. Kato, Tilting complexes defined by idempotents, Comm. Algebra 30(1) (2002), 83-100.

10. L. Kadison, New examples of Frobenius extensions, University Lecture Series, 14 (American Mathematical Society, Providence, RI, 1999).

11. L. Kadison, Separability and the twisted Frobenius bimodule, Alg. Rep. Theory 2 (1999), $397-414$.

12. H. Krause, Smashing subcategories and the telescope conjecture - an algebraic approach, Invent. Math. 139(1) (2000), 99-133.

13. H. Minamoto and I. Mori, The structure of AS-Gorenstein algebras, Adv. Math. 226(5) (2011), 4061-4095.

14. J. Miyachi, Derived categories with applications to representation of algebras. Personal notes. Available at http://www.u-gakugei.ac.jp/ miyachi/papers/ChibaSemi.pdf, accessed 2 May 2012.

15. K. Morita, Adjoint pairs of functors and Frobenius extensions, Sci. Rep. Tokyo Kyoiku Daigaku Sect. A, 9 (1965), 40-71.

16. A. Neeman, The Grothendieck duality theorem via Bousfields techniques and Brown representability, J. Amer. Math. Soc. 9 (1996), 205-236.

17. A. Neeman, Triangulated categories, Annals of Mathematics Studies, vol. 148 (Princeton University Press, Princeton, NJ, 2001).

18. C. Serpé, Resolution of unbounded complexes in Grothendieck categories, J. Pure Appl. Algebra, 177 (2003), 103-112. $121-154$

19. N. Spaltenstein, Resolutions of unbounded complexes, Compositio Math. 65 (1988), 